\title{
Two decades after Human Genome Project: do large-genetic studies lead to path of the genomic medicine of complex diseases?
}

\author{
Yoichiro Kamatani ${ }^{1,2} \cdot$ Yukinori Okada ${ }^{30}{ }^{3,4}$ \\ Received: 3 August 2020 / Accepted: 3 August 2020 \\ (c) The Author(s), under exclusive licence to The Japan Society of Human Genetics 2020
}

"First, do no harm." is said to be one of the well-known sentences from the Hippocratic Oath. Genetic medicine inherently violates this principle since it may invoke genetic discrimination, or it could bring serious problems to the life of the family of the individual with heritable pathogenic variant. Genomic medicine can be justified only if it can contribute significantly to better health outcome. Genomic medicine for rare genetic disorders and cancer have already got consensus for their clinical utility, but how about complex diseases?

The very first genome-wide association study for complex disease was reported in 2002 by Ozaki et al., soon after the determination of the draft human genome sequence by the International Human Genome Project. Since then, the era of large human genetic studies was opened, and so many advances followed, including the development of genomewide SNP genotyping array and next generation sequencer, the construction of large genetic consortium and national biobanks, and the introduction of several sophisticated statistical genetics analyses. These developments led to effective genome-wide association analysis (GWAS) as a tool to elucidate genetic architectures of complex diseases.
Rapid development of epigenomic- and transcriptomicsequencing technologies, including a recent break-through of single cell sequencing, further enables comprehensive biological interpretation of the results of GWAS. GWAS can also be regarded as an experimental validation of Ronald A Fisher's theoretical polygenic model proposed in 1918. As a consequence, it strongly contributed to the understanding of the relationship between genetics and human complex traits. Polygenic risk score (PRS) is used to predict the occurrence of complex diseases. Although it could not prove its utility at early phase, current PRS for complex disease using more than thousands of genetic variants yields prediction accuracy equivalent with monogenic diseases.

Now it is high time to think about the possibility of utilizing genomic information in a clinical practice of complex diseases. We invite young and established leaders in genetics, most of them are active researchers in Japan, to discuss about large genetic studies and its future perspectives. We hope this collection of papers contribute to equip the readers for the era of genomic medicine for complex diseases.
Yoichiro Kamatani

kamatani.yoichiro@edu.k.u-tokyo.ac.jp

1 Laboratory of Complex Trait Genomics, Graduate School of Frontier Sciences, the University of Tokyo, Tokyo, Japan

2 Laboratory for Statistical and Translational Genetics, RIKEN Center for Integrative Medical Sciences, Yokohama, Japan

3 Department of Statistical Genetics, Osaka University Graduate School of Medicine, Osaka, Japan

4 Laboratory of Statistical Immunology, Immunology Frontier Research Center (WPI-IFReC), Osaka University, Suita, Japan 